Cahiers d'études japonaises

19 | 2012

Le Japon et le fait colonial II

\title{
Claire-Akiko Brisset, Arnaud Brotons et Daniel Struve (dir.), De l'épopée au Japon : narration épique et théâtralité dans le Dit des Heike
}

\section{Florence Goyet}

\section{(2) OpenEdition}

Journals

Édition électronique

URL : https://journals.openedition.org/cipango/1719

DOI : $10.4000 /$ cipango.1719

ISSN : 2260-7706

Éditeur

INALCO

Édition imprimée

Date de publication : 30 octobre 2012

Pagination : 217-221

ISBN : 978-2-85831-204-7

ISSN : $1164-5857$

Référence électronique

Florence Goyet, «Claire-Akiko Brisset, Arnaud Brotons et Daniel Struve (dir.), De l'épopée au Japon: narration épique et théâtralité dans le Dit des Heike », Cipango [En ligne], 19 | 2012, mis en ligne le 06 mai 2014, consulté le 30 juin 2021. URL : http://journals.openedition.org/cipango/1719; DOI : https:// doi.org/10.4000/cipango.1719

Ce document a été généré automatiquement le 30 juin 2021.

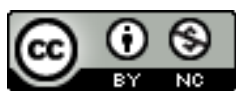

Cipango est mis à disposition selon les termes de la Licence Creative Commons Attribution - Pas d'Utilisation Commerciale 4.0 International. 


\title{
Claire-Akiko Brisset, Arnaud Brotons et Daniel Struve (dir.), De l'épopée au Japon : narration épique et théâtralité dans le Dit des Heike
}

\author{
Florence Goyet
}

\section{RÉFÉRENCE}

Claire-Akiko Brisset, Arnaud Brotons et Daniel Struve (dir.), De l'épopée au Japon : narration épique et théâtralité dans le Dit des Heike, Paris, Riveneuve, Collection « Actes académiques - série japonaise », 2011, 205 pages + illustrations hors texte. ISBN : 978-2-36013-069-6.

1 La nouvelle collection de Riveneuve s'adresse à la fois aux spécialistes et au grand public. Pari superbement tenu avec cet ouvrage, qui donne les actes d'un colloque de juin 2008 à l'Université Paris VII (plus une communication tenue en 2010 à Paris sur un thème proche). Les huit textes sont très érudits et devraient pourtant intéresser « tous les lecteurs curieux du Japon, sa société, sa culture et ses arts ", à qui la nouvelle collection de Riveneuve est aussi dédiée. À l'occasion de l'exposition Hiroshige ${ }^{1}$, on pouvait ainsi voir des piles de l'ouvrage sur les tables de la librairie de la Pinacothèque de Paris.

Les deux premiers articles s'intéressent à la légende de Miminashi Hōichi, bien connue en Occident depuis que Lafcadio Hearn en a fait un conte. Claire Brisset (« À propos de la récitation épique : la légende de Miminashi Hōichi ») reconstitue les maillons de la chaîne qui a pu mener des récits oraux au texte du Gayū kidan de 1782, source de Hearn. Tout en fournissant au lecteur non spécialiste les éléments du dossier, même les plus connus, elle donne un aperçu des différents textes, et établit un tableau comparatif de différentes versions imprimées ou diffusées. Elle rappelle l'importance de la récitation 
en temps de troubles, "cadre général » dans lequel «il faut resituer la légende de Miminashi Hōichi » (p. 41). Le centre d'intérêt de C. Brisset, dans la droite ligne de ses travaux précédents et de ceux de Florence Dupont, est en effet le cadre rituel de la récitation, la légende de Miminashi Hōichi illustrant le « danger de la performance hors du rituel » (p. 48).

La communication de Hiromi Hyōdō («Les moines joueurs de biwa [biwa hōshi] et le Dit de Heike ») se construit sur une série d'approches successives, des caractéristiques du biwa de Satsuma à la tradition shamanique dont relevaient les récitants, et au regard d'Artaud qui a donné sa propre version du conte - lumineusement mise en perspective. Hyōdō en vient à définir le moine au biwa, cet être à l' "identité floue » (p. 68), comme l'intermédiaire possible non seulement entre morts et vivants, mais aussi entre le tumulte ineffable du «monde naturel » et l'homme "dévasté par le logos " (Artaud, cité p. 60). Miminashi Hōichi, le récitant aveugle et qui sera sans oreilles, est ainsi capable de se mettre en résonance avec « le tumulte des existences invisibles » (p. 62), et de répondre à la voix « comme exhalée de l'essence des choses » (Artaud ; p. 60).

4 Arnaud Brotons («Les sanctuaires de Kumano dans le Dit des Heike: un lieu pour les morts?») déploie dans toute sa profondeur la problématique des rapports entre les Taira et un sanctuaire marginal et pourtant essentiel. Lointain, d'accès malaisé, mais considéré comme une " antichambre donnant accès à l'autre monde» (p. 73), Kumano «éclipse les autres lieux» (p. 76), attirant empereurs retirés et aristocratie, bientôt accompagnés d'une foule d'humbles si nombreux qu'on en venait parfois à les refouler. A. Brotons fait le lien avec le grand bouleversement de l'époque: en mettant « massivement en scène » ces « nouveaux dieux » au détriment du sanctuaire impérial, le Heike reflèterait leur surgissement dans le paysage religieux (p.76), mais aussi la «nouvelle source de légitimité » qu'ils représentent, que le Heike développe en même temps que le chaos du pays (conclusion de l'article, p. 90 sq.). La deuxième partie développe le lien profond et circonstancié avec les Taira. L'ascension de Kiyomori lui est attribuée. A. Brotons développe l'épisode de la carpe de mer, et son ambiguïté (la carpe est consommée par Kiyomori, dans un contexte d'interdits alimentaires stricts). Le dieu de Kumano, dieu de la vie et de la mort - par l'intermédiaire historique des moines qui passent aux Minamoto (p. 88 sq.) - amène aussi la perte des Taira, en raison de leurs « forfaits » (développement sur le rôle de Shigemori, parallèle à celui qu'il joue dans le Heiji - de façon peut-être plus intéressante d'ailleurs que dans le Heike -, puis sur la noyade de Koremori, annonciatrice de celle d'Antoku).

Komine Kazuaki et Daniel Struve s'intéressent tous deux au texte même du Heike, et à ses transformations entre les différentes versions. Komine («La version dite Amakusabon du Dit des Heike, et la récitation ») décrit cette version inclassable, rédigée dans un style dialogué par un Japonais, Fukan Habian (Fabien), à la demande des Jésuites. Après avoir présenté l'auteur et ses écrits - de sa conversion au catholicisme à son abjuration et son Contre Dieu -, il décrit l'Amakusa-bon (1592), version abrégée, en langue moderne et en caractères romains. L'article montre qu'en condensant les récits, Habian supprime de fait toute une série d'éléments bouddhiques pourtant essentiels au texte, donnant ainsi naissance à une "version aberrante", récriture "par et pour des chrétiens» (p. 107). D'autre part, il montre l'importance profonde de la forme dialoguée choisie par l'auteur, qui fait du Heike monogatari un zōdan 䧱談, forme dont Komine a déjà donné des analyses. Le zōdan, "particulièrement en vogue au Moyen Âge » (p. 101), permet une grande énergie. Il a aussi pour intérêt de nous fournir une 
série de notations très précieuses, qui « reflètent très concrètement les conditions et l'atmosphère de la récitation " épique, et construisent finalement une "performance idéale » (p. 103).

Daniel Struve ("Récits de fin : narration épique et scénicité ») s'intéresse au saigo, ce récit des derniers instants d'un guerrier, considéré comme un " morceau de bravoure " par les joueurs au biwa. Malgré la grande diversité des épisodes, on peut « considérer que le saigo constitue bien un motif récurrent", et qu'il est lié en profondeur aux représentations que les guerriers ont de leur condition (p. 113-114). À partir de là, l'article regarde de près trois exemples dans deux versions éloignées, la version «à lire » Engyō-bon et la version " à réciter » Kakuichi-bon. La comparaison précise amène à conclure que le Kakuichi-bon privilégie constamment la dimension scénique, réorganisant les épisodes et modifiant « le rôle et le caractère » des personnages « dans le sens de l'exemplarité » (p. 116). Vraisemblance psychologique et historique s'effacent au profit d'une « cohérence essentiellement scénique» (p.117), et d'une «idéalisation de la figure du guerrier $»$.

7 Les deux communications suivantes sont liées par le thème du passage à la forme théâtrale. Stanca Scholz-Cionca («Les nō guerriers et le Dit des Heike: procédés de transposition ») analyse les procédés de ce qui lui paraît une véritable distanciation (au sens brechtien): mise en abyme des épisodes guerriers, restructuration autour de quelques images-leitmotive, et renversement de perspective -qu'elle décrit précisément dans Nue. Le nō déconstruit et reconstruit les récits, suivant une logique propre, comme il reconstruit les visages par les masques. Créant des personnages « aux identités multiples et instables » (p. 134), il montre que leur être véritable n'est pas la forme quotidienne qu'ils revêtent dans le début de la pièce, mais bien le spectre. La chronologie narrative est abandonnée, les échanges se déploient dans une autre temporalité, et le texte se centre autour d'images poétiques fortes - S. Scholz-Cionca développe avec bonheur celle du bois mort et de la pirogue dans Sanemori et dans Nue. La conclusion est que finalement, dans de tels cas, le Heike ne joue plus qu'un rôle « subordonné » (p. 144).

8 Il en joue un moindre encore dans les kinpira-jōruri analysés par Jean-Jacques Tschudin («Le Kinpira jōruri : héros épiques dans le théâtre de poupées d'Edo»). Fleurissant au début de la période d'Edo, ces pièces ont pour héros des personnages qui renvoient à une époque bien plus ancienne que les faits du Heike: fin du $x^{e}$ siècle pour la première génération de ces shitennō, ces « braves aux capacités hors du commun, voire carrément surhumaines » (p.151). Après avoir rappelé la situation du théâtre de poupées à Edo et le succès monumental des histoires de Kinpira, de ses père et oncles, cousins et descendants -, Tschudin décrit le répertoire, l'accent mis sur les liens féodaux, et le possible rôle thérapeutique de telles histoires après le grand incendie d'Edo en 1657. Plus profondément, il évoque les dilemmes auxquels se trouvent confrontés les héros, déchirés entre désir de se réaliser comme individu et désir d'être "simultanément reconnu comme membre du groupe en acceptant d'en suivre les règles » (p. 161). Plus encore qu'à l'incendie, l'apparition de ces pièces aurait répondu au bouleversement d'un changement de monde et à la difficulté à trouver sa place dans « la société civile, apaisée, qui s'installe » (p. 161).

9 L'article de Rainier Lanselle («Prendre la parole : le théâtre du Bord de l'eau ») porte sur la littérature chinoise. Il analyse le rôle essentiel et paradoxal d'une forme de théâtre, le zaju ("théâtre varié »-la «forme reine du théâtre au XIV siècle », p. 177), dans la 
constitution du roman Au Bord de l'eau. Quatre éléments sont soulignés : 1/ Le zaju opère le passage du récit du conteur à la récitation à la première personne, véritable «bond historique» (p.178), et qui s'accompagne d'une subjectivation soulignée par le texte. 2/ Le rapport à l'oralité est essentiel, et la langue vernaculaire s'étend (R. Lanselle donne des tableaux détaillés, des exemples abondants et précis de cette évolution). 3/ Les zaju, tout en utilisant le même matériel narratif, ne sont pas superposables au roman, et ne reprennent pas les épisodes qui se retrouveront dans le roman. "Récits périphériques", "amplifications partielles autour de tel ou tel personnage", ils auraient été élaborés en marge du cœur de la tradition, qui, lui, « aurait été l'apanage de conteurs qui s'en transmettaient la tradition de façon plus ou moins réservée " (p. 196 sq.). 4/ Du point de vue thématique, cette "prise de parole» apporte une "structure de discours" qui va permettre une subversion plus intime. La langue vulgaire porte la mutation sociale à l'œuvre, elle vient dire le destin qui bascule, la nécessité d'être un bandit pour être loyal.

Si l'on peut faire une objection à ce livre, elle porterait au fond uniquement sur son titre, sans doute choisi par la maison d'édition. Le sujet commun à ces études, présent en filigrane derrière presque tous les articles, serait plutôt l'idée de "récitation ». En l'appelant De l'épopée, on annonce une réflexion d'ensemble sur le genre épique. Mais le lecteur qui espérerait y trouver une réflexion sur l'épopée au Japon restera sur sa faim. Peut-être cela tient-il à une orientation qui est souvent celle des chercheurs japonais (par exemple Kusaka Tsutomu, 2008²), pour qui les gunki monogatari ne peuvent pas être rapprochés des épopées du reste du monde. Cela tient peut-être aussi à une filiation avec les thèses de Florence Dupont, pour qui le texte même de l'épopée ne saurait guère avoir d'intérêt. On a quelques allusions de-ci de-là (à l'épique selon Bakhtine ${ }^{3}$, à un ouvrage pédagogique général sur la littérature médiévale ${ }^{4}$, ou à l'inévitable Homère et Dallas ${ }^{5}$ ), mais pas de réflexion sur le Heike comme épopée ni sur ce que peut être l'épopée au Japon ${ }^{6}$. L'épopée est plutôt ici un corpus qu'un problème. Cela n'ôte rien à l'intérêt d'un ouvrage toujours passionnant.

\section{NOTES}

1. Pinacothèque de Paris, du 3 octobre 2012 au 17 mars 2013.

2. Kusaka Tsutomu 日下力, Ikusa monogatari no sekai : chūsei gunki bungaku o yomu, いくさ物語の 世界: 中世軍記文学を読む, Tōkyō, Iwanami shoten, 2008.

3. Mikhail M. Bakhtine, Esthétique et théorie du roman, Paris, Gallimard, 1978.

4. Nelly Labère, Littératures du Moyen Âge, Paris, PUF, collection «Licence ", 2009. Aurait été plus topique une référence à mon propre livre : Penser sans concepts: fonction de l'épopée guerrière: Iliade, Chanson de Roland, Hōgen et Heiji monogatari, Paris, Champion, 2006.

5. Florence Dupont, Homère et Dallas: introduction à une critique anthropologique, Paris, Hachette, 1990.

6. Est typique de cette perspective la remarque de R. Lanselle au début de son article : «Ces deux romans, que la sinologie américaine désigne souvent, non sans raison, du terme d'épopées (...) ». Ni références, ni discussion. 


\section{AUTEUR}

\section{FLORENCE GOYET}

Université Stendhal-Grenoble III 